\title{
Recent Trends in Research and Development on Environmental Engineering
}

\author{
Masanobu IIDA \\ Environmental Engineering Division
}

\begin{abstract}
Wayside environmental problems in railways largely involve noise, ground vibration and tunnel micro-pressure wave. This review describes the results of recent studies into these issues conducted by Railway Technical Research Institute.
\end{abstract}

Keywords: wayside environment, noise, ground vibration, tunnel micro-pressure wave

\section{Introduction}

Wayside environmental problems largely involve noise, ground vibration and tunnel micro-pressure wave. These problems can be regarded as oscillatory and acoustic phenomena in physics. The May 2015 issue of RTRI Report featured "environmental engineering," and included seven papers on the latest research and development in the field of matters relating to the wayside environment. Oscillatory and acoustic phenomena are ubiquitous dynamic phenomena, present in various technical railway areas. As such, RTRI Report has thus far presented many studies on wayside noise and vibration, not only featuring "environmental engineering" but also through "railway dynamics," and "materials technology," etc. To help readers grasp recent trends in research and study related to the wayside environment at RTRI, this review paper presents work on the wayside environment that has been published in RTRI Report over the past two years or so.

\section{Wayside noise}

Studies on wayside noise look at subjects such as: evaluating the contribution of individual and separate noises such as rolling noise, aerodynamic noise and bridge noise to total noise; identifying the characteristics and mechanisms generating individual noises; developing methods to abate noise and its transmission; and developing methods to estimate wayside noise at the planning stage of new lines or when contemplating higher running speeds. The results of recent RTRI studies on each of these areas are summarized below.

\subsection{Evaluation of individual noise contribution to total noise}

"Contribution of Rolling Noise and Aerodynamic Noise to the Total Noise Generated from the Lower Part of Shinkansen Cars Running at High-speed" [1]

It is now known that for Shinkansen vehicles, a significant share of the noise measured at $25 \mathrm{~m}$ above the ground when the cars are running at $200 \mathrm{~km} / \mathrm{h}$ or more, is generated by the current collection system and from the lower parts of the vehicles. Although lower body part noise is caused by both rolling noise and aerodynamic noise coming from the bogies etc., the percentage contribution to total noise of these separate noise sources was hitherto unclear, which prevented the development of specific noise reduction measures. As such, this paper evaluates the contribution of each of these noises to total lower body part noise by measuring total noise during on-track tests and numerical analyses of rolling noise, and obtained the following results:

- For vehicles running at $315 \mathrm{~km} / \mathrm{h}$ or lower, the greatest source of the total noise from lower body parts of the cars was rolling noise while aerodynamic noise was generated in the frequency range at and below $1600 \mathrm{~Hz}$.

- For vehicles running at $335 \mathrm{~km} / \mathrm{h}$ or higher, the upper limit of the frequency range of aerodynamic noise rose to $3150 \mathrm{~Hz}$ making it account for at least $50 \%$ of the total noise.

\subsection{Evaluation of individual noise source character- istics}

(1) "Vibration Characteristics of a Wheel of a Running Railway Vehicle” [2]

While both rails and wheels need to be investigated to identify the rolling noise characteristics, the study discussed in the paper looked specifically at the vibration of wheels by conducting experiments and calculations. First, vibration characteristics of a stationary wheel were examined by impact vibration testing. This was followed by measurement of the vibration characteristics of a wheel under a running vehicle using accelerometers mounted on the wheel. The results were then compared; it was found that the peak frequency of the wheel under vibrational acceleration in the running test in both radial and axial directions roughly agreed with the peak frequency of the stationary wheel under radial vibration in the vibration test and that the vibration velocity level of both the tire and web of the wheel of the running vehicle followed the cubic law of the vehicle running speed.

(2) "Analytical Study on Structure Member Vibration Characteristics of Reinforced Concrete Rigid Frame Viaduct” [3]

Numerical analyses were conducted and on-track measurements were taken to identify the noise characteristics of reinforced concrete rigid frame viaducts when vehicles were running at $300 \mathrm{~km} / \mathrm{h}$ or higher. First, a new numerical analysis method was proposed whereby the entire largescale, complex railway system was divided into a vehicle/ track system and a track/structure system for efficient calculation. With the vehicle/track system, the vibrating load of a running train was calculated by analyzing the interac- 
tion of the vehicle/track system taking into account high frequency components of track irregularity. With the track/ structure system, structural vibrations were analyzed with reduced degrees of freedom using the modal method. Second, using the method, vibration analyses were conducted systematically changing various parameters on typical reinforced concrete rigid frame viaducts. The results of these analyses showed that the following factors played a dominant role in bridge noise generation: axle arrangement at and below $20 \mathrm{~Hz}$, unsprung mass and long wave track irregularity from 20 to $100 \mathrm{~Hz}$, rail fastening intervals from 100 to $150 \mathrm{~Hz}$ and track irregularity at and above $150 \mathrm{~Hz}$.

(3) "Improvement of Measurement Methods for Aeroacoustic Noise from Bogie Section in the Wind Tunnel Test" [4]

With Shinkansen trains, noise generated by the current collection system and noise generated by lower body parts (especially the bogies) accounted for a significant portion of the total aerodynamic noise. Since the development of the first Shinkansen train, the number of current collecting units per train set has drastically dropped while the number of bogies per train set has not changed. In order to mitigate wayside noise to allow Shinkansen trains to run faster in the future, reducing lower body part noise is a priority. A low-noise wind tunnel is typically used for experiments on aerodynamic noise. When such experiments are carried out, it is desirable to ensure that the inflow of air through the model bogie in the experiment reflects the corresponding inflow of real cars as much as possible. In the study discussed in this paper, on-track tests were performed to measure the vertical distribution of the axial component of flow velocity at the center of the track in order to grasp the flow velocity distribution approaching the bogie. In the next step of the study, using the results of the above measurement as the conditions for the inflow, a wind tunnel test method was developed to assess aerodynamic noise from the bogie. Finally, using the method, aerodynamic noise from the bogie was measured. From the measurements, it was found that the accuracy in shape of the bogie model used in the experiment affected noise spectra.

\subsection{Noise mitigation measures}

(1) "Improvement of Noise Insulation Material for a Rail Joint" [5]

Sound insulation material for rail joints that can be easily installed on site was developed and then improved, primarily for electrically insulated joints on conventional lines with long welded rails. As an earlier prototype was found to not offer satisfactory noise mitigation performance on commercial lines, this original design was improved by adding a $100 \mathrm{~mm}$-thick sound insulation panel above the original noise insulation material. The improved prototype was then subjected to on-track tests. In addition, the improved prototype was tested together with track noise insulation bags filled with crushed stones and rail noise insulation material which had been developed earlier for ordinary sections without joints. It was found that, by combining these three materials, noise from the power cars and trailers, both measured near the rails, dropped by about $3 \mathrm{~dB}$ and $3.5 \mathrm{~dB}$ respectively.

(2) "Reduction of Aerodynamic Noise Generated by a Pantograph Head by Using Speaker-driven Synthetic Jet Ac-

\section{tuators" [6]}

RTRI has been conducting a wide range of basic research and development to reduce aerodynamic noise from pantograph heads through flow field control techniques. This paper investigated the possibility of using speakerdriven synthetic jet actuators (SJA), known for efficiently controlling flow fields, to reduce pantograph head noise. In the study, CFD analysis found that Karman vortices can be reduced most efficiently by locating jet outlets at the lower corner on the side of the upstream end of the head. To verify the CFD analysis, wind tunnel tests were conducted using a two-dimensional head model on which 10 outlet holes were provided at the lower corner on the side of the upstream end of the model and 10 SJAs were then embedded in these holes. The test found that aeolian sound could be reduced at a wind velocity of $90 \mathrm{~km} / \mathrm{h}$. It was also found that, by driving speakers adjoining one another in an opposite phase rather than in a coordinate phase, the speaker driving noise could be reduced while aeolian sound was also kept to a minimum.

(3) "A Basic Study of Aerodynamic Noise Reduction Method of a Pantograph Head Using Plasma Actuators" [7]

In addition to the SJA solution described above to reduce aerodynamic noise through flow field control, plasma actuators (PAs) were subjected to wind tunnel tests and CFD analyses for noise reduction effect. Wind tunnel tests in the low velocity range found that flow separation could be reduced by PAs, weakening Karman vortices down the stream. Subsequent CFD analyses found that, with enough output power available, PAs should be effective in controlling the flow field around the head, thus reducing aerodynamic noise.

(4) "A Study on Aerodynamic Noise Reduction Method of a Pantograph Head Using Flow Control Techniques" [8]

Following the PA and SJA trials described above, a steady suction technique was investigated for a possible third solution to reduce aerodynamic noise from the pantograph head through flow field control. Using this technique, attempts were made to reproduce the PA's flow field control in the high velocity range. Wind tunnel tests verified that, providing steady suction near the separation line around the head could weaken Karman vortices and thus reduce aeolian sound. In addition, more detailed CFD analyses and wind tunnel tests were conducted on SJAs, in which it was found that more efficient flow field control was possible by making the SJA outlets rectangular in shape and changing their aspect ratio, thus modifying the characteristics of vortices generated by SJAs.

(5) "Reducing Effect of Absorbing Materials Covering the Inner Walls of the Tunnel Entrance Hood on Noise from a Tunnel Portal of Shinkansen" [9]

The wayside noise generated by Shinkansen trains passing through tunnel portals consists of running noise normally generated in the open section; and noise that is generated by trains running inside tunnels, which is then reverberated through the tunnel and finally emitted from the portal. The latter noise must also be mitigated. Entrance hoods installed at Shinkansen tunnel portals are designed to reduce tunnel micro-pressure waves. In the study discussed here, quantitative acoustic experiments were conducted using a $1 / 20$ th scale model of the entrance hood, whose inner walls were covered with sound absorb- 
ing material, to find out whether the modification would have any noise mitigation effect. It was found that, with the upper (top or sloped) part of the entrance hood covered with sound absorbing material, the level of noise emitted through the portal measured at a point lower than the rail level (RL) dropped. Subsequent measurement of noise in field tests with entrance hoods covered with the sound absorbing material, produced similar results, indicating that the noise mitigation technique is also effective in practice.

\subsection{Noise prediction method}

"Study on a Prediction Model for Wayside Noise of Shinkansen in Cut Sections" [10]

The distribution of wayside noise in cut sections is influenced by a range of factors including noise reflected off the cut walls. Using this premise, experiments were conducted using an acoustic model to look into noise distribution in cut sections. Based on the results of that process, improvements were made to the existing Shinkansen noise prediction method for cut sections. The experiment showed that the distribution of wayside noise in cut sections was strongly influenced by sound reflecting off the cut's walls and that the sound was strongly reflected diagonally and upward. In addition, a new prediction model that factors in sound reflected off cut walls was introduced in the existing noise prediction method, and comparisons were made between the noise prediction method and the acoustic model experiment. The result was that the prediction method was fairly accurate with cut walls of $5 \mathrm{~m}$ or less in height.

\section{Ground vibration}

The study on ground vibration covers the following topics: clarifying the physical characteristics and mechanisms of ground vibration caused by running trains; developing vibration mitigation methods, and developing methods for predicting ground vibration on new lines and with increased train speeds.

(1) "Propagation Characteristics of Horizontal and Vertical Component of Train-induced Ground Vibration" [11]

To predict and take measures against ground vibrations along Shinkansen lines, it is essential to identify the propagation characteristics of ground vibrations including their horizontal motion. This study investigated the characteristics of the horizontal and vertical motions of ground vibrations along Shinkansen lines based on the results of excitation tests and field vibration measurements conducted in the past. It was found that the normalized spectra of components at the same locations were almost unchanged even with different excitation conditions involving train speed, axle arrangement and other parameters, showing that excitation force characteristics had only a small impact on ground vibration propagation characteristics. It was also found for both vertical and horizontal motions that ground vibration attenuation occurred relatively close to structures and that structural types and ground conditions had a major impact on, among other things, the intensity of ground vibration and attenuation distance.

(2) "The Influence of the Variation of Track Support Rigidity Around an Overbridge on Ground Vibration" [12]
The area near an abutment of an overbridge in an embankment section has structural borders between the embankment, abutment and girder. The area is also where ballast support rigidity changes. Furthermore, the area is where the embankment tends to loosen, causing exciting forces to change rapidly. This study investigated the influence of changes in exciting forces near an abutment on ground vibrations, based on the combination of dynamic analyses of the train-track system and the structureground system. Investigations were also conducted on the effect of vibration mitigation work for overbridges. It was found that exciting forces increased behind embankments around abutments and that exciting forces increased in proportion to looseness or settlement of the area behind embankments. The study also showed that wayside ground vibrations were lessened around the natural frequency of the overbridge girder through vibration mitigation measures such as increasing the overbridge girder thickness and installing dampers at the ends of the girder.

(3) "Improvement of the Low-temperature Characteristics of Resilient Rail Pads by Foam Structuring” [13]

While being effective in mitigating ground vibration and bridge noise, track pads tend to have poorer damping performance at low temperature as the pads' modulus of elasticity increases. In order to improve low-temperature characteristics of low-spring-constant track pads, attempts were made to improve the material and structure of ethylene propylene diene monomer (EPDM). In the attempts, the rubber material was made into foam while the shape of the pad was changed from a grooved structure into flat plates. The result was an improved distribution of stress. Air trapped in the foamed rubber structure is expected to absorb impact load, thus improving damping performance. In addition, a foamed version of the current material, i.e. styrene butadiene rubber (SBR), was produced, partly aiming at cost reduction of the material. The prototype was then subjected to impact tests using a weight to evaluate its damping performance. Both of those new materials were found to have significantly improved low-temperature characteristics over the current material.

\section{Tunnel micro-pressure wave}

The study on tunnel micro-pressure waves covers the following topics: clarifying the physical characteristics and generation mechanism of micro-pressure waves; developing micro-pressure wave mitigation methods, and developing methods for predicting micro-pressure waves on new lines and with increased train speeds.

(1) "Development of Experimental Technique on the Micropressure Wave Phenomenon Using a Train Model of Actual Shape" [14]

In order to clarify tunnel micro-pressure wave phenomena and develop methods to predict and mitigate the phenomenon, it is important to accurately analyze compression waveforms generated when a train enters a tunnel. Model experiments are generally used as a method to evaluate compression waves. In this study, a model train with the same geometry as an actual train and launching method were developed to conduct model experiments which could be used with the existing rotating wheel-type 
launcher. The method makes it possible to conduct experiments using model train at a maximum speed of around $250 \mathrm{~km} / \mathrm{h}$. Experiments using the method found the following: experiments using axisymmetric model trains offered sufficient levels of accuracy when there was no significant flow separation at train nose; and that the accuracy of evaluation in model experiments could be improved by using actual-shape model trains when there was significant flow separation at the train nose caused by parts with sharp edges such as snow ploughs and skirts.

(2) "Experimental Investigation of the Effects of Topography Around the Tunnel Portal on the Micro-pressure Wave" [15]

The spatial distribution of waveforms and peak values of tunnel micro-pressure waves can vary depending on the topography of the surrounding area. In this study, a model experiment was conducted using a model train launcher to examine the influence of topographical conditions on the radiation of tunnel micro-pressure waves. In the experiment, the spatial distribution of the peak value of tunnel micro-pressure wave was studied for three topographical conditions around a tunnel portal: cut land on one side, cut land on both sides and a viaduct. It was found that with cut land on one side, the peak value of micro-pressure wave on the ground was higher as the height of the cut land was greater and that the value was lower on the cut land than on the ground. In addition, based on the results of the measurements, parameters representing topographic influence were established.

(3) "Micro-pressure Wave Emitted from a Tunnel Hood with Side Openings" [16]

The tunnel "entrance" hood with side openings at the portal is a primary method for mitigating tunnel micropressure waves. With the Shinkansen's double track tunnels shared by the up and down lines, a tunnel entrance hood on the "opposite" line is located at the "exit" portal (on the micro-pressure wave radiation side) of the tunnel. In this study, the impact of the tunnel "exit" hood on micropressure wave radiation was examined. Model experiments found, among other things, that the larger the total area of the side openings on the tunnel exit hood, the greater the peak values of the micro-pressure wave were around the openings compared to ordinary tunnel portals. It was also found that it was possible to produce similar results of the model experiments by acoustical analysis.

(4) "Countermeasure for Reducing Micro-pressure Wave Emitted from Railway Tunnel by Installing Hood at the Exit of Tunnel" [17]

As described above, with the Shinkansen's double track tunnels, a tunnel "entrance" hood on the opposite line is in effect at the tunnel "exit" portal, affecting the characteristics of the micro-pressure wave emitted from the tunnel exit. In this study, the tunnel entrance hood on the opposing line was regarded as a hood at the tunnel exit, or the micro-pressure wave radiation side of the tunnel, and measures for adding micro-pressure wave mitigation features to the exit hood were considered. Specifically, A new concept hood was designed with an inner wall in its crown forming a space in the longitudinal direction between the crown and the inner wall, (hereafter referred to as the "inner wall" ) closed at the end towards the exit and open at the end facing into the tunnel. (The hood is hereaf- ter referred to as "the exit hood with an inner wall." ) To examine micro-pressure wave mitigation effect of the exit hood with an inner wall, acoustical calculations and model experiments were conducted. It was found, among other things, that the new design produced micro-pressure wave mitigation effect corresponding to the ratio between the cross-sectional area of the space between the crown and the inner wall to the total cross-sectional area of the hood, and that the results of acoustical calculations agreed fairly well with the results of model experiments.

\section{Conclusion}

Railway operators are continuing to work towards higher train running speeds and larger transport capacity on both Shinkansen and conventional lines, and therefore mitigation of wayside environmental impacts is becoming increasingly important. RTRI will continue to conduct research and development on wayside environment and would appreciate continued support and cooperation from all involved.

\section{References}

[1] Kitagawa, T., et al., "Contribution of Rolling Noise and Aerodynamic noise to the Total Noise Generated from the Lower Part of Shinkansen Cars Running at Highspeed," RTRI Report, Vol.27, No.1, pp.23-28, 2013 (in Japanese).

[2] Murata, K., et al., "Vibration Characteristics of a Wheel of a Running Railway Vehicle," RTRI Report, Vol.27, No.1, pp.35-40, 2013 (in Japanese).

[3] Watanabe, T., et al., "Analytical Study on Structure Member Vibration Characteristics of Reinforced Concrete Rigid Frame Viaduct," RTRI Report, Vol.27, No.10, pp.47-52, 2013 (in Japanese).

[4] Yamazaki, N., et al., "Improvement of Measurement Methods for Aeroacoustic Noise from Bogie Section in the Wind Tunnel Test," RTRI Report, Vol.27, No.1, pp.29-34, 2013 (in Japanese).

[5] Hansaka, M., et al., "Improvement of Noise Insulation Material for a Rail Joint," RTRI Report, Vol.28, No.2, pp.23-28, 2014 (in Japanese).

[6] Sato, Y., et al., "Reduction of Aerodynamic Noise Generated by a Pantograph Head by using Speaker-driven Synthetic Jet Actuators," RTRI Report, Vol.26, No.8, pp.5-10, 2012 (in Japanese).

[7] Mitsumoji, T., et al., "A Basic Study of Aerodynamic Noise Reduction Method of a Pantograph Head using Plasma Actuators," RTRI Report, Vol.27, No.10, pp.1116, 2013 (in Japanese).

[8] Mitsumoji, T., et al., "A Study on Aerodynamic Noise Reduction Method of a Pantograph Head Using Flow Control Techniques," RTRI Report, Vol.28, No.12, pp.11-16, 2014 (in Japanese).

[9] Kawaguchi, T., et al., "Reducing Effect of Absorbing Materials Covering the Inner Walls of the Tunnel Entrance Hood on Noise from a Tunnel Portal of Shinkansen," RTRI Report, Vol.28, No.3, pp.11-16, 2014 (in Japanese). 
[10] Ogata, Y., et al., "Study on a Prediction Model for Wayside Noise of Shinkansen in Cut Sections," RTRI Report, Vol.28, No.3, pp.5-10, 2014 (in Japanese).

[11] Yokoyama, H., et al., "Propagation Characteristics of Horizontal and Vertical Component of Train-induced Ground Vibration," RTRI Report, Vol.25, No.11, pp.3540, 2011 (in Japanese).

[12] Kato, S., et al., "The Influence of the Variation of Track Support Rigidity Around an Overbridge on Ground Vibration," RTRI Report, Vol.28, No.3, pp.17-22, 2014 (in Japanese).

[13] Suzuki, M., et al., "Improvement of the Low-temperature Characteristics of Resilient Rail Pads by Foam Structuring," RTRI Report, Vol.28, No.2, pp.17-22, 2014 (in Japanese).
[14]Fukuda, T., et al., "Development of Experimental Technique on the Micro-pressure Wave Phenomenon Using a Train Model of Actual Shape," RTRI Report, Vol.27, No.1, pp.5-10, 2013 (in Japanese).

[15] Miyachi, T., et al., "Experimental Investigation of the Effects of Topography Around the Tunnel Portal on the Micro-pressure Wave," RTRI Report, Vol.28, No.3, pp.23-28, 2014 (in Japanese).

[16] Atsumi, H., et al., "Micro-pressure Wave Emitted from a Tunnel Hood with Side Openings," RTRI Report, Vol.28, No.3, pp.29-34, 2014 (in Japanese).

[17]Saito, S., et al., "Countermeasure for Reducing Micropressure Wave Emitted from Railway Tunnel by Installing Hood at the Exit of Tunnel," RTRI Report, Vol.27, No.1, pp.17-22, 2013 (in Japanese).

\section{Author}

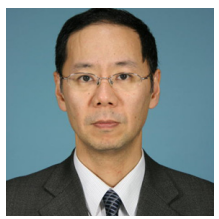

Masanobu IIDA, Dr. Eng.

Principal Researcher, Environmental

Engineering Division 